\title{
Influence of stress on the field emission properties of amorphous carbon thin films and multiwall carbon nanotube- polymer composites
}

\author{
${ }^{a)}$ C. H.P Poa, R. G. Lacerda ${ }^{*}$ and S.R.P. Silva \\ Advanced Technology Institute, School of Electronics and Physical Sciences, University of Surrey, \\ Guildford GU2 7XH, United Kingdom \\ F. C. Marques \\ Universidade Estadual de Campinas, Unicamp, Instituo de Fisica "Gleb Wataghin" -13083-970, \\ Campinas-SP, Brazil
}

Amorphous carbon thin films are unique semiconducting films whose electronic and mechanical properties are determined by the $\mathrm{sp}^{2}$ and $\mathrm{sp}^{3}$ hybridizations. Recent work has shown that the field emission properties from amorphous carbon films containing nano-crystalline particles (NAC) can be dependent on its intrinsic stress and its local density [1]. This behaviour in NAC films is believed to be attributed to the modification of the local electronic properties of graphitic nanocrystal when subjected to high stress. Carbon nanotubes on the other hand are graphitic tubes with high aspect ratio and interesting electronic properties. Single walled nanotubes can be metal or semiconducting depending on its chirality, where multiwall nanotubes (MWNT) are generally metallic. Reports have shown that by applying pressure on nanotubes its electronic properties can be modified where its band structure varies with stress.

In this work, the NAC films and MWNT composite thin films were subject to external stress by mechanical bending. Compressive stress was applied to the samples using a three-point bending technique (Figure 1). The samples were bent using an optical fibre placed in the middle of the sample and two glass slides were used to clip the sample at both ends to create a curvature. Optical fibres ranging from $50 \mu \mathrm{m}$ to $1000 \mu \mathrm{m}$ were used to allow different compressive stresses to be applied on the samples. Field emission properties were measured using the probe technique. The anode is placed directly above the region with the curvature where the stress is expected to be maximum.

Three a-C films with low (1 GPa), medium $(6.5 \mathrm{GPa})$ and high intrinsic stress $(11.7 \mathrm{GPa})$ was used to represent films. For the MWNT composite films, undoped MWNTs with $8 \%$ wt. concentration were mixed with polystyrene (PS) and hotpressed to form cathodes. The emission threshold fields of a MWNT-PS sample experiencing different applied stress loadings varied from 2.3 to $0.7 \mathrm{~V} / \mu \mathrm{m}$ before rising back to $3.2 \mathrm{~V} / \mu \mathrm{m}$ when the applied stress to the film was removed (Figure 2). This result is consistent with the results observed from NAC films where the applied stress modified the field emission properties. This study is further compared to the field emission results from a-C films with different intrinsic stress. Figure 3 shows the field emission threshold fields of a-C films with different intrinsic stress. The bending of the low stress film shows a very interesting sequence of results, as shown in figure 3 (a). Upon the application of external stress, $E_{h}$ initially at $37 \mathrm{~V} / \mu \mathrm{m}$ falls to $24 \mathrm{~V} / \mu \mathrm{m}$, as the amount of stress is increased due the change in the curvature. This reduction of $\mathrm{E}_{\mathrm{th}}$ we believe is due to the increase of internal stress by having an external

Engineering Department, University of Cambridge, Trumpington Street, Cambridge, CB2 1PZ, United Kingdom 
compressive force applied on the film. As the curvature is further increased, the stress becomes too high to be sustained, and the threshold field increases. Hence, $E_{\text {th }}$ increases to $44 \mathrm{~V} / \mu \mathrm{m}$ as a maximum stress is exceeded. This can be attributed to the high intrinsic stress within the films, not being exceeded by the external pressure on the film or the phenomenon responsible for the electron density/decrease in threshold exceeding the "stress threshold value". In summary, this work investigates the effects of stress on carbon-based materials during field emission.

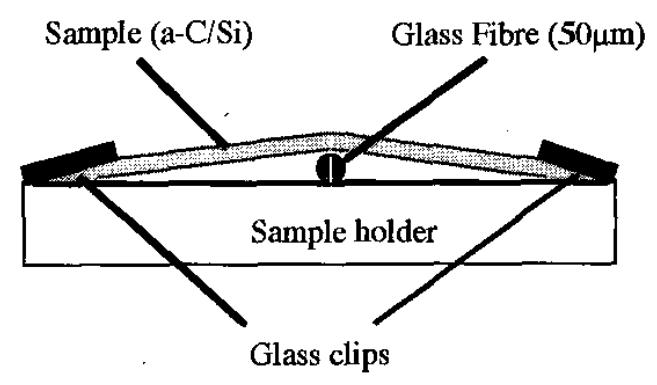

Figure 1. 3-point bending setup

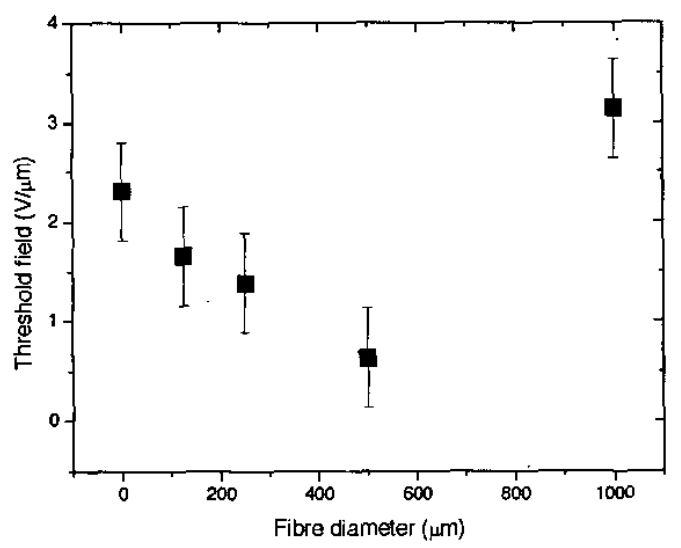

Figure 2. Field emission threshold field of MWNTPS sample as a function of the glass fibre diameter.

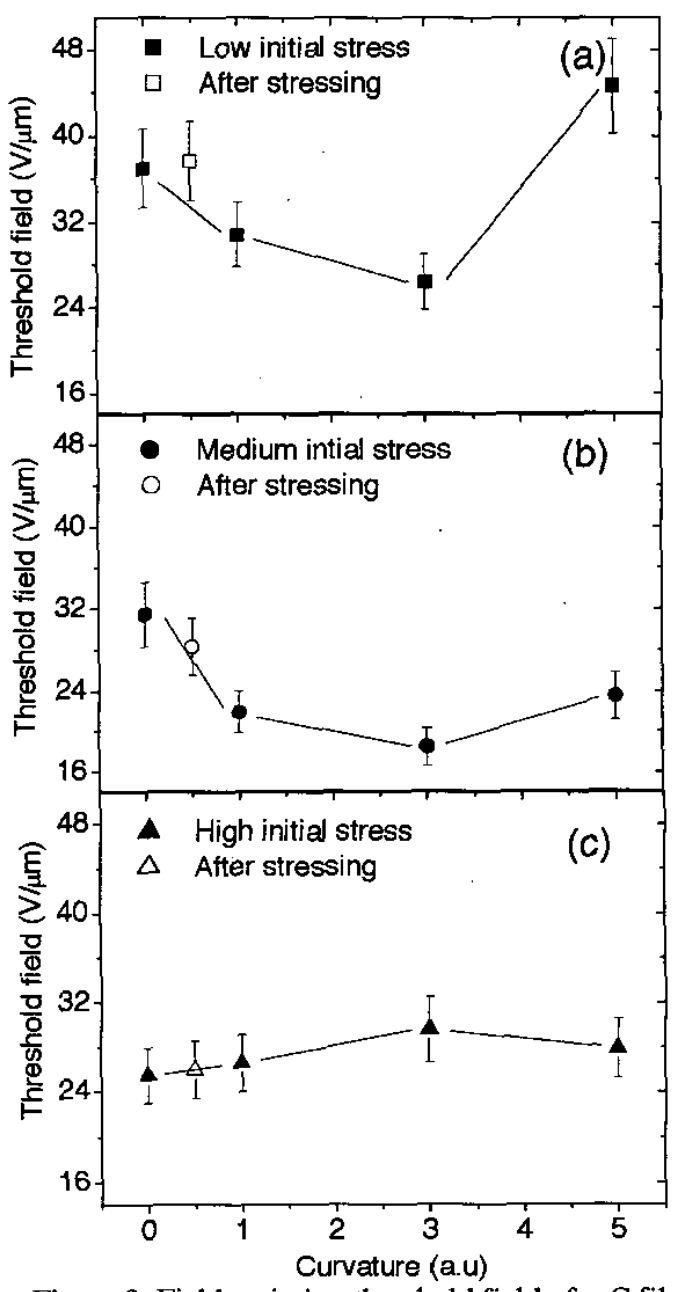

Figure 3. Field emission threshold field of a-C films as a function of the curvature due to bending.

Dr C. H. P. Poa

E-mail: Patrick.poa@eim.surrey.ac.uk

[1] C. H. Poa, R. G. Lacerda, D. C. Cox, S. R. P. Silva, and F. C. Marques Appl.

Phys. Lett. 81, 853 (2002) 